Monatsschr Kinderheilkd 2013 · 161:499-499

DOI 10.1007/s00112-012-2831-1

Online publiziert: 25. Mai 2013

(c) Springer-Verlag Berlin Heidelberg 2013

\section{F. Zepp ${ }^{1} \cdot$ B. Willershausen ${ }^{2}$}

${ }^{1}$ Zentrum für Kinder- und Jugendmedizin, Universitätsmedizin, Johannes-Gutenberg-Universität Mainz

2 Poliklinik für Zahnerhaltungskunde und Parodontologie,

Universitätsmedizin, Johannes-Gutenberg-Universität Mainz

\title{
Zahn- und Mundgesundheit
}

Die Möglichkeiten zur Krankheitsprävention und Gesundheitsförderung im Bereich der Zahnmedizin konnten insbesondere in den letzten 20 Jahren deutlich aufgezeigt werden, und die konsequente Realisierung entsprechender Prophylaxekonzepte führte nachweislich zu großen Erfolgen. Gerade bei Kindern und Jugendlichen müssen Präventionsmaßnahmen frühzeitig beginnen, um spätere Gesundheitsschäden und auch, besonders im Bereich der Zahn- und Mundgesundheit, Beeinträchtigungen der Lebensqualität und teure zahnärztliche Behandlungen zu vermeiden.

Eine lebenslange Mundgesundheit kann erreicht werden, wenn bereits im frühen Kindesalter Prophylaxemaßnahmen eingeübt und konsequent beibehalten werden. Dazu gehören richtiges Ernährungsverhalten, effektive Mundhygienemaßnahmen, eine kindgerechte Zahnputztechnik und die frühzeitige Nutzung von Fluoriden. Ein weiterer wichtiger Pfeiler für die Erhaltung der Zahngesundheit ist die bewusste, zuckerarme Ernährung. RegelmäBige zahnärztliche Kontrolltermine sowie Gruppen- oder Individualprophylaxemaßnahmen ermöglichen das frühzeitige Erlernen von Verhaltensmustern zur Erhaltung der Zahngesundheit. Eine besondere Bedeutung kommt auch der Diagnostik möglicher Kiefer- und Zahnfehlstellungen zu, da eine rechtzeitige Therapie im Milchund frühen Wechselgebiss Fehlfunktionen und Wachstumsbeeinträchtigungen verhindert.

Trotz der guten Ergebnisse der präventiven Maßnahmen und des allgemeinen Rückgangs der Karies zeichnet sich in Deutschland eine Polarisierung der frühkindlichen Karies ab. Ein Großteil der Kinder und Jugendlichen weisen heutzutage naturgesunde Gebisse auf; Kinder aus Familien mit risikoorientierten Verhaltensweisen zeigen dagegen häufig ein überdurchschnittlich hohes Kariesrisiko.

\section{I) Kinderärzte spielen bei der Früherkennung von Zahnschäden eine entscheidende Rolle}

Kinderärzte begleiten die Entwicklung des Kindes und Jugendlichen, ihnen fällt damit zwangsläufig die Rolle des ersten Blicks und der Überweisung zum Zahnarzt und/oder Kieferorthopäden zu. Ziel der Leitthemenbeiträge des vorliegenden Heftes ist es, alle relevanten Bereiche der präventiven Zahnmedizin darzulegen und auch Hinweise zur Therapie entsprechender Erkrankungsformen im Mundbereich einschließlich der Korrektur von Zahnfehlstellungen zu geben. Insbesondere soll das multifaktorielle Geschehen der frühkindlichen Karies aufgezeigt werden, um Kinderärzte zur Früherkennung dieses Krankheitsbildes zu sensibilisieren. Wir hoffen, dass diese Beiträge zum Verständnis und zur Wachsamkeit für mögliche beginnende Erkrankungszeichen im Mundbereich sowie von Zahnfehlstellungen und den Bedarf für erforderliche kieferorthopädische Behandlungen beitragen und wertvolle Hinweise für die kinderärztliche Tätigkeit geben.

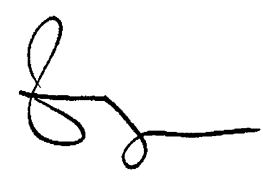

Prof. Dr. Fred Zepp

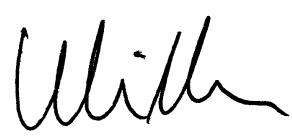

Prof. Dr. Brita Willershausen

\section{Korrespondenzadresse}

Prof. Dr. B. Willershausen

Poliklinik für Zahnerhaltungskunde und Parodontologie, Universitätsmedizin, Johannes-Gutenberg-Universität Mainz Augustusplatz 2, 55131 Mainz willersh@uni-mainz.de 